\title{
Space Surveillance And Early Warning Radars: Buried Treasure For The Information Grid
}

\author{
Charles P. Satterthwaite \\ AFRL/IFTA \\ Bldg. 620, 2241 Avionics Circle, WPAFB, Ohio 45433-7334 \\ DSN 785-6548 x3584, COM 937-255-6548 x3584, FAX 937-656-4277 \\ charles.satterthwaite@wpafb.af.mil
}

\begin{abstract}
A major emphasis of the modern Air Force is to drastically improve the capability of the Global Information Grid, also called the Global Grid. It is believed that the realization of this capability will greatly enhance the Dynamic Aerospace Command's desire to be able to be Globally Aware, be able to Globally Engage its forces, and also be able to make Dynamic Decisions. The expectation of the Global Grid is that it will be able to provide accurate, secure, and timely information to our commanders anywhere, anytime, and in their specific information application requirement.
\end{abstract}

A strong emphasis has been placed on the new technologies needed to achieve this capability. Many of the Defense Advanced Research Project Agency's (DARPA) new research priorities have been focused at improving interoperability, information assurance, information accessibility, and new space based information platforms to accommodate the evolving Global Grid.

There is a resource for the Global Grid that should not be forgotten. That is the Nation's investment in Space Surveillance and Early Warning Ground Based Radar Systems. These systems where originally built to keep a constant watch for the real threat of nuclear missile attack throughout the world. The strong requirements for these systems provided an enormous capability to monitor air and deep space activities.

The Aerospace Command and Control and Intelligence, Surveillance, and Reconnaissance (C2ISR) Campaign Plan 2000 has a focus plan called Global Information Grid: The focus plan states that we need to provide a Global Information Grid infrastructure to obtain seamless, protected, reliable, worldwide connectivity to support all C2ISR mission needs. Of particular near-term interest are improvements in terrestrial and space infrastructures for "outside the gate" needs and fixed deployed and airborne infrastructures for "inside the gate" information needs. We must also procure, maintain and upgrade "information appliance", both wired and non-wired, to achieve common, interoperable, scaleable components that enable plug-and-play into the Global Information Grid.

This paper will explore how the Air Force's valuable investments in Space Surveillance and Early Warning Ground Based Radar Systems can be expanded upon to enable the Global Information Grid. 


\section{The Infosphere And The Global Information Grid}

The easiest way to describe the Infosphere is to think of it as the Internet In The Sky. The Internet connects anyone with a computer, a modem, and a few software applications to the growing domain of electronic commerce. The amount of information available over the Internet, and its accessibility to growing numbers of consumers is largely responsible for the rigorous growing information based economy. Consumers can now validate product and service claims through alternate sources, and can increasingly carry out electronic transactions.

The Infosphere is an expansion of the Internet. The Internet is largely dependent on telecommunication networks. Internet users are plugged into a single source network. This network is primarily two dimensional, though satellite data links greatly enhance the speed and access sites of the network. The Infosphere is multi-dimensional. Infosphere users are plugged into multiple information sources, including the Internet, which are time tagged, integrated, and filtered to give expanded real-time (or near real-time) solutions. Besides the Internet, information sources include direct data links with air, ground, and sea weapon systems in the area of interest, on-line databases and knowledge bases, and on-line experts. Time tagging assures the integrity and linking of information sources. Integration of information is taking the parts of different sources to arrive at a specific solution. Filtering information is necessary to weed out redundant or obsolete information from the desired solution.

The Global Information Grid is the projection of the Internet, as the Infosphere, completely around the Earth, giving access of all available information to everyone, anytime, everywhere. The growing realization of the Global Information Grid and the Infosphere requires increasing attention to Information Security. This is because individuals and organizations must have some level of privacy to guarantee their rights, business practices, and their secrets.

\section{The Joint Battlespace Infosphere}

The executive summary of the United States Air Force Scientific Advisory Board Report on "Building the Joint Battlespace Infosphere" (1) defines the Joint Battlespace Infosphere (JBI) as a combat information management system that provides individual users with the specific information required for their functional responsibilities during crisis or conflict. The JBI integrates data from a wide variety of sources, aggregates this information, and distributes the information in the appropriate form and level of detail to users at all echelons. The JBI was originally described in the 1998 USAF Scientific Advisory Board (SAB) report Information Management to Support the Warrior. At the joint task force (JTF) commander's level, the JBI is a powerful command and control (C 2) system that combines inputs from a variety of sources, including existing $\mathrm{C} 2$ systems, reconnaissance data, satellite data, unit capability data, logistics data, and real-time battlefield conditions. The JBI builds an aggregated picture from these combined inputs, giving unparalleled situational awareness accessed as easily as a web page. The JBI also provides for speedy downward flow of information, so when commanders order an 
action, the action is received and implemented at the subordinate level almost immediately. The commander in chief (CINC) or JTF commander creates a JBI for a specific purpose, usually in response to a crisis or conflict. The JBI enables the commander to focus information support for a specific operational purpose, ensure or limit access to critical information, and provide an information management system that can respond to natural or enemy actions that disrupt communications capabilities. As units are assigned to the mission, their information needs are electronically identified, and available information is automatically accessed. Thus, deployed units are ready to fight immediately upon being deployed or assigned (1).

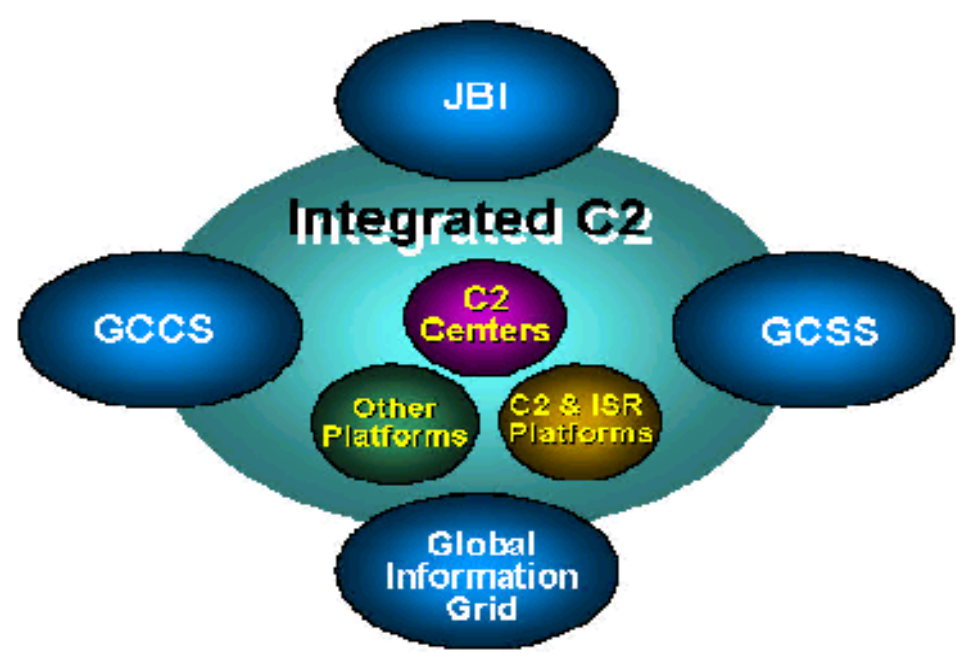

Figure 1 - Integrated Command and Control

Supporting these capabilities and forming a foundation of the JBI is a platform of protocols, processes, and common core functions that permit participating applications and organizations to share and exchange critical mission information in a timely manner. It provides uniform rules for publishing new and updated objects into the JBI and promptly alerts any JBI clients that have subscribed to such objects. These properties enable dynamic information flows among client programs of the JBI, serving to integrate the clients to conduct a single mission. The JBI platform integrates many individual information systems that currently support operational forces. Each existing system has been developed in a stove-piped fashion; few interoperate with each other. The JBI acts as an intermediary between these systems, converting information from one representation to another to enable interoperability. In addition to acting as middleman between disparate systems, the JBI interprets the information flowing between applications, using it to build its own, more complete, picture of the current situation. Furthermore, the JBI tailors this picture for individual users: the commander gets a highlevel view of the campaign, while the soldier in the field gets a detailed description of a nearby hostile base. The JBI provides an architecture for the incorporation of future data capture technologies that exploit better sensors, databases, fusion engines, automated analysis tools, collaborative planning and execution aides, and distribution controls. It is also a disciplined process that guides the activities of people responsible for obtaining, 
verifying, fusing, presenting, analyzing, and controlling the information necessary for success in any operation (1).

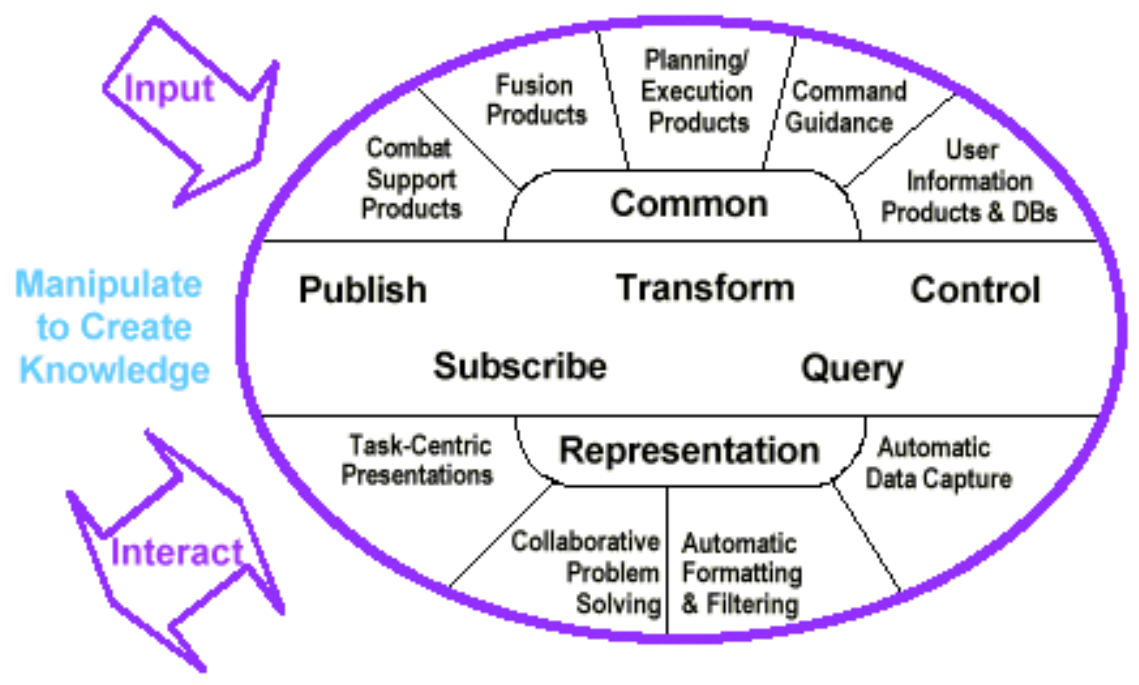

Figure 2 - The JBI Functionality

The JBI is connected to, and interoperable with, a variety of existing and planned $\mathrm{C} 2$ and combat support information systems. The JBI is not intended to replace $\mathrm{C} 2$ systems, but to be the substrate for integrating them. The JBI subscribes to pertinent information published by supporting systems and, when necessary, pulls specific information from other networks. In addition, the JBI connects to fusion engines and may perform fusion on its own, thereby ensuring that the most complete and coherent picture of the battlefield situation resides within the JBI itself. The JBI concept recognizes that display technology is constantly advancing and that new displays must be tailored for users from flight leader to JTF commander. The JBI provides services through a federation of multiple servers. The Global Information Grid connects these servers to each other and to the many systems that support the JBI. Many of the servers provide services from the rear via reach-back, thereby limiting the forward footprint of the JBI (1). 


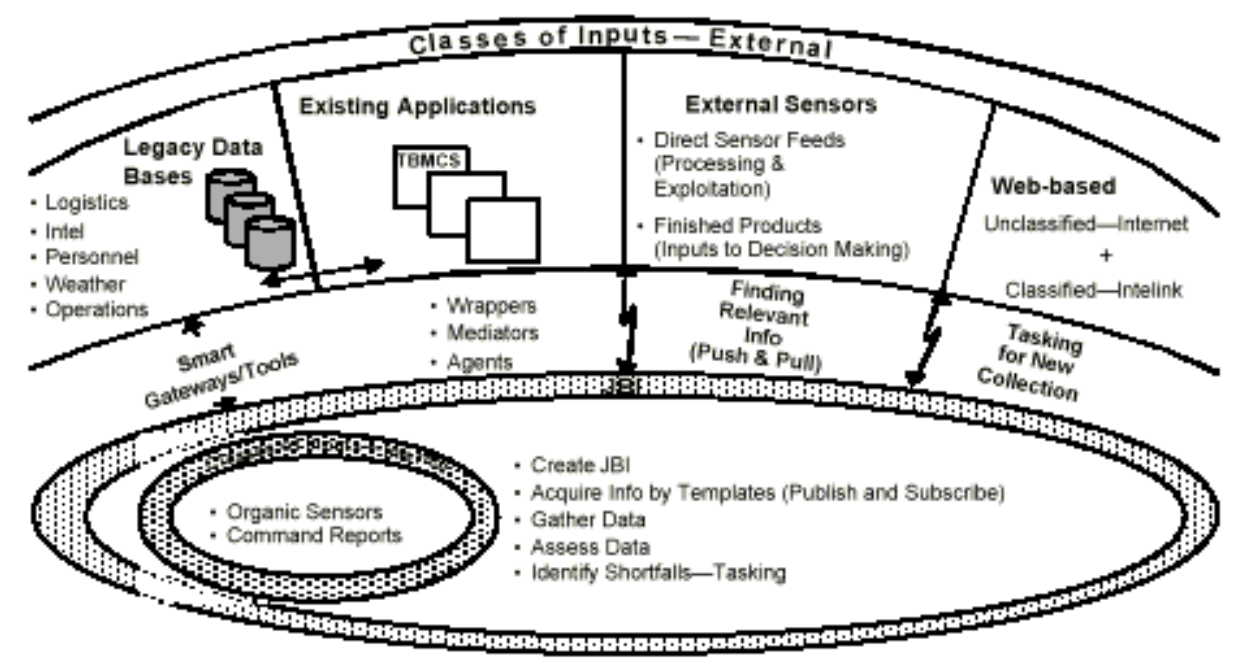

Figure 3 - The JBI Architecture

\section{Sources of Information}

The Defense Advanced Research Projects Agency (DARPA) initiated several programs in the early 1990s to address the Nations growing dependence on Intelligent Integrated Information (I3). A DARPA Broad Area Announcement (BAA) called I3 dealt with the problem of pulling together heterogeneously distributed information from databases, knowledge bases, and other sources into a useful solution to complex user queries. Some of the programs of $\mathrm{I} 3$ dealt with intelligent query processing, information extraction, information classification, object exchange modeling, and temporal solution sets.

The need for these types of information sciences stemmed from increasing dependence of defense and civilian organization on ballooning information resources. Many organizations have ample in-house information resources (or access to them) to perform their many functions. The problem is the shear volume and complexity of these growing resources. Combining local expertise with historical archives and current market trends to answer a customer request for service must happen quickly. Several specialty businesses arose in the late 1970s and 1980s to address these information service requests with dedicated information service applications. These applications specialized in obtaining, updating, and integrating specialized information in a timely manner. But these selfcontained services were complex and very expensive. Only large, well-established organizations could afford them. A new demand was created by small and upstart organizations that also needed these same services.

The I3 programs provided an enormous increase to information and a cultural shift away from dedicated super-solutions. Many of the popular query processing engines available on the Web is in some way, related to the I3 researchers. One example of an I3 program is illustrated in Figure 4, the Heterogeneous Integration of Processed Engineering Design (HIPED). HIPED provides a means to combine existing knowledge of engineering design archives, expand those designs with new database elements, and assess the outcome. 


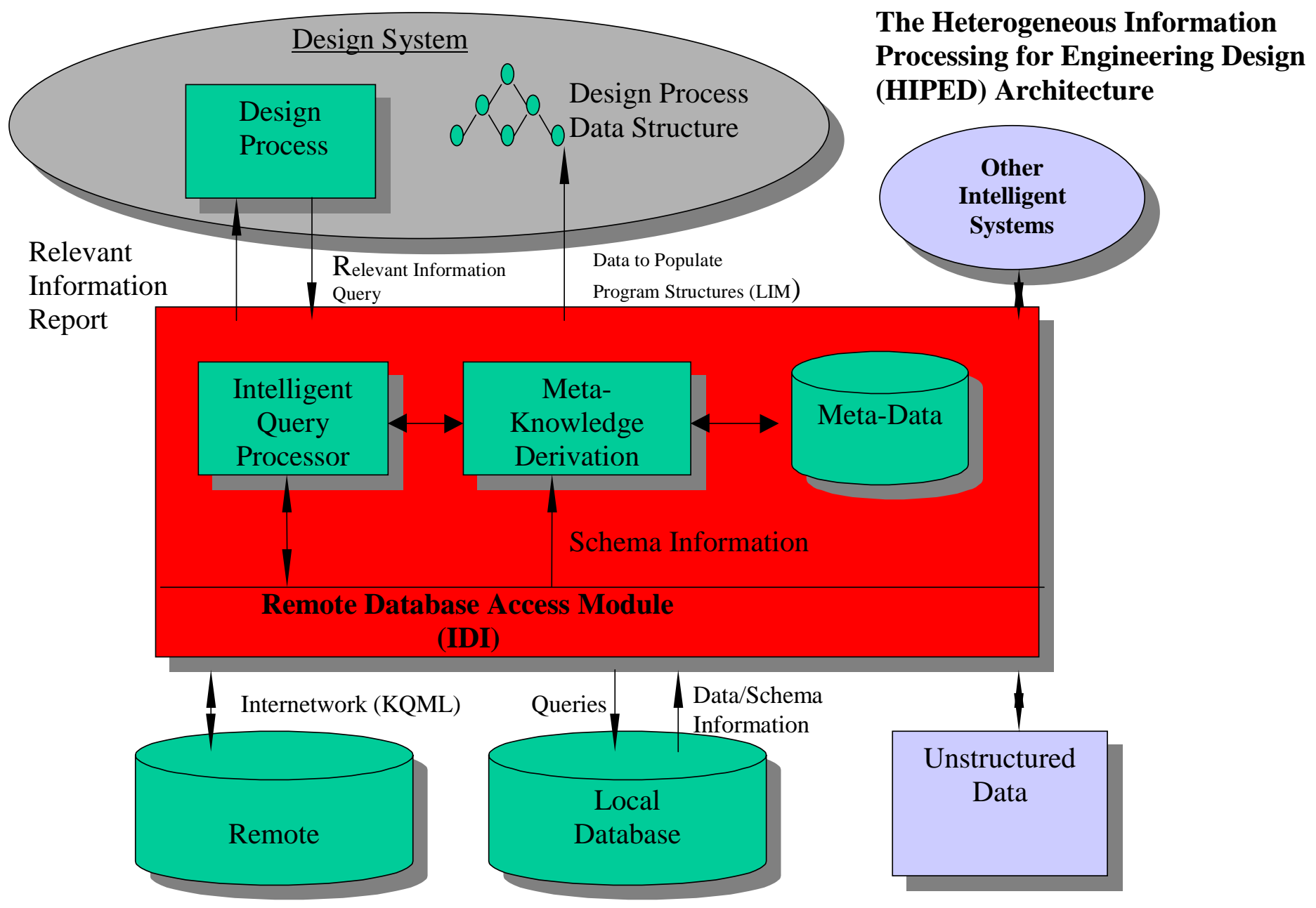

Figure 4 - Heterogeneous Integration of Engineering Design 
An important concept to grasp when trying to understand the information age is that all information has sources. The information flows from the source. It is the source that must be understood, and facilitated in order to benefit from its information. The structure of the source, the properties of the source, the temporal nature of the source information must be understood in order to exploit it. A key concept of the I3 program was that it sought to utilize source information with minimal changes to the source infrastructure. In many cases, this can be accomplished by interfacing the source and the requestor with services that transparently convert and integrate sources outputs to the requestor's format. It also transparently converts the requestor's queries into acceptable formats for the sources. These services are a series of wrappers and mediators. Wrappers are non-intrusive layers of services around information sources, which format source inputs and outputs, without altering the source structure or functionality. Mediators are services between the sources and users that provide specific services such as filtering, sequencing, integrating, appending, interpreting, converting, or whatever redundant activity a user may require.

Sources of information become the key ingredient to the integrity of any informationbased system. The quality and accuracy of information is key to making effective decisions. An information source doesn't have to be the newest and best technology; it can be from the most ancient archived historical record. An excellent approach to understanding the information domain requirements is to do a detailed study of the available information sources. It could very well be that what is held in hand could be the most potent for getting the job done

\section{Space Surveillance Radars and Early Warning Radars as Information Sources}

This paper proposes that Space Surveillance and Early Warning Radars are excellent sources of information needed for Global Information Grid Infrastructure. It was stated earlier that: "Of particular near-term interest are improvements in terrestrial and space infrastructures for "outside the gate" needs and fixed deployed and airborne infrastructures for "inside the gate" information needs. We must also procure, maintain and upgrade "information appliance", both wired and non-wired, to achieve common, interoperable, scaleable components that enable plug-and-play into the Global Information Grid." A readily available source of solutions to this directive is the Space Surveillance Radars and Early Warning Radars.

\section{A Brief History of Early Warning and Space Surveillance Radars}

The following historical summary comes from the Air Force Space Command's (AFSPC) Homepage. In September 1957, the United States and Canada created the North American Air Defense Command (NORAD), the first bi-national, joint-service military command on the North American continent. This new defense partnership was responsible for air defense of the combined airspace of the United States and Canada. Air Defense Command was the Air Force component of Continental Air Command (CONAD), the United States component of NORAD. In October 1960, NORAD's mission changed with the assumption of operational control of all space defense responsibilities with the formation of the Space Detection and Tracking System (SPADATS). CONAD maintained administrative control of SPADATS. In 1963, the Air 
Force began work on the world's first phased array radar, the AN/FPS-85, at Eglin AFB FL. Expected to enter service in 1965; this powerful spacetrack system was designed to provide tracking data on thousands of space objects per day. However, a fire, which destroyed the entire system, and technical problems, delayed the system's final acceptance until 1967. The system achieved initial operational capability (IOC) in 1969. Technological advancement in the late 1950s and early 1960s enabled the U.S. and Soviet Union to develop sea-launched ballistic missiles (SLBMs). To provide timely warning against Soviet Sea Launched Ballistic Missiles (SLBMs), the Air Force began work on an interim SLBM detection network consisting of several AN/FSS-7 radars located on the Atlantic, Pacific and coasts. The network, eventually controlled by the 4783rd Surveillance Squadron of the 14th Aerospace Force, was fully operational by May 1972. By July 1975, the AN/FPS-85 radar at Eglin AFB had been reprogrammed to provide additional SLBM detection and warning capability along with its original spacetrack mission. Seeking to improve and enlarge its SLBM detection capability, the Air Force began the development of a new phased array radar system called AN/FPS-115 PAVE PAWS in August 1973. The first PAVE PAWS at Otis ANGB, MA became operational in April 1980. The next two operational PAVE PAWS sites were established at Beale AFB, CA and Robins AFB, GA. The fourth site at Eldorado AFS, TX became operational in May 1987. Currently, only PAVE PAWS radars located at Cape Cod Air Force Station, Mass., and Beale AFB; California are operational.

Since the late 1950s, the U.S. military had sought to create an anti-ballistic missile (ABM) system to guard against the Soviet missile attack that seemingly loomed on the horizon. Although many systems were designed, only Safeguard ABM reached operational status. The Army closed their only operational Safeguard ABM site near Grand Forks AFB, North Dakota in February 1976 after Congress objected to its high cost and questionable effectiveness. The following year the Air Force acquired the unused advanced phased array radar at the site near Concrete, ND for use in SLBM and ICBM warning. This new radar, the AN/FPQ-16 Perimeter Acquisition Radar Characterization System (PARCS), provided SLBM warning over the Hudson Bay and additional coverage of the central BMEWS area as well as extremely accurate space surveillance data.

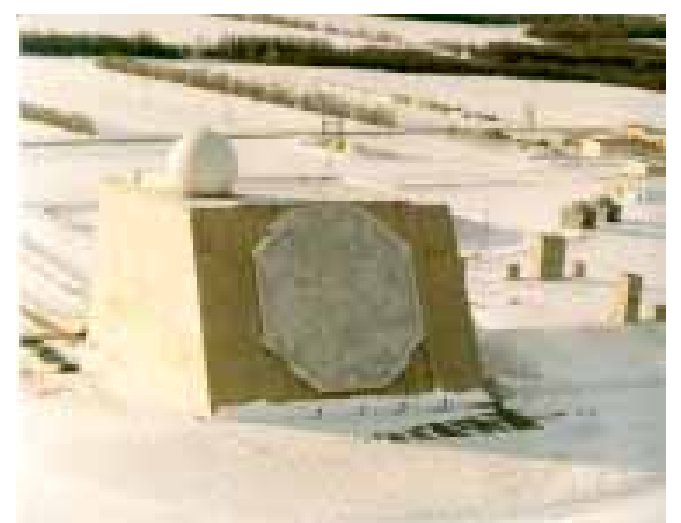

Figure 5 - PARCS Early Warning Radar 


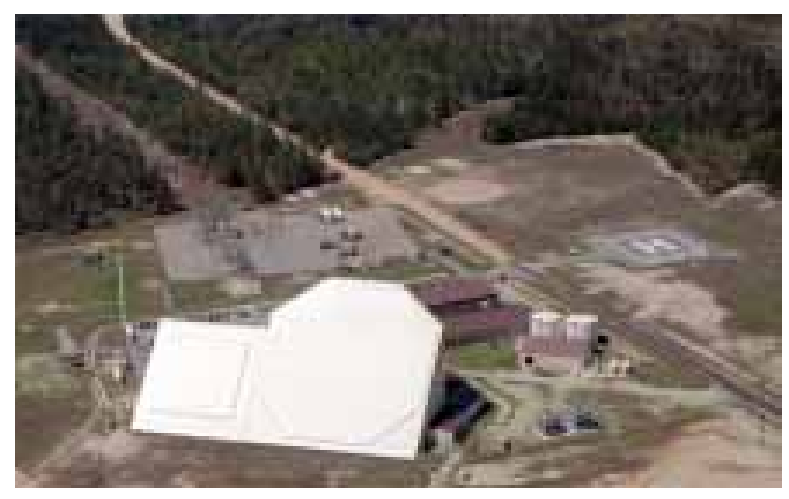

Figure 6 - Eglin AFB Space Surveillance Radar

PAVE PAWS is an Air Force Space Command radar system operated by four 21st Space Wing squadrons for missile warning and space surveillance. PAVE PAWS radars are located at Cape Cod Air Force Station, Mass., and Beale AFB; Calif. PAVE is an Air Force program name, while PAWS stands for Phased Array Warning System. The radar is used primarily to detect and track sea-launched (SLBM) and intercontinental ballistic missiles (ICBMs). The system also has a secondary mission of Earth-orbiting satellite detection and tracking. Information received from the PAVE PAWS radar systems pertaining to SLBM/ICBM and satellite detection is forwarded to the U.S. Space Command's Missile Warning and Space Control Centers at Cheyenne Mountain Air Station, Colo. Data is also sent to the National Military Command Center and the U.S. Strategic Command.

The unique aspect of the PAVE PAWS radar system is the phased array antenna technology. This system differs from mechanical radars, which must be physically aimed at an object in space to track or observe it. The phased array antenna is in a fixed position and is part of the exterior building wall. Phased array antenna aiming, or beam steering, is done rapidly by electronically controlling the timing, or phase, of the incoming and outgoing signals. Controlling the phase through the many segments of the antenna system allows the beam to be quickly projected in different directions. This greatly reduces the time necessary to change the beam direction from one point to another, allowing almost simultaneous tracking of multiple targets while maintaining the surveillance responsibility. The large fixed antenna array through its better beam focusing, improves system sensitivity and tracking accuracy. 


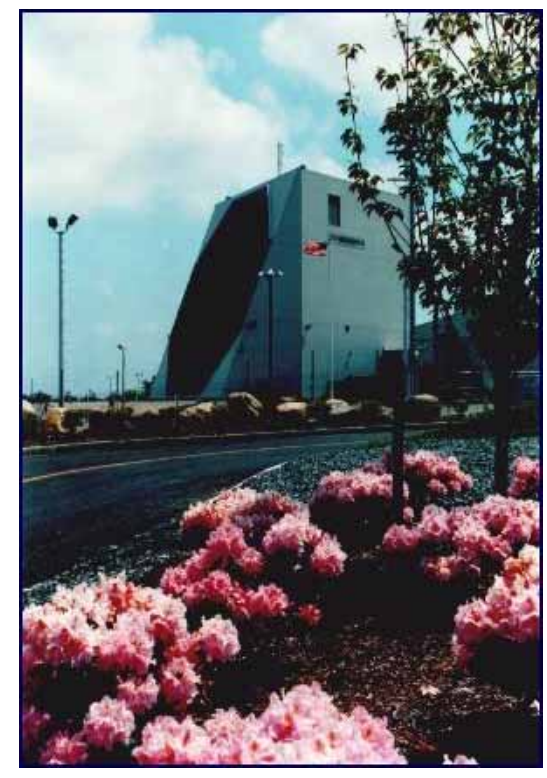

Figure 7 - PAVE PAWS Early Warning Radar

A phased array antenna, as any other directional antenna, will receive signals from space only in the direction in which the beam is aimed. The maximum practical deflection on either side of antenna center of the phased array beam is 60 degrees. This limits the coverage from a single antenna face to 120 degrees. To provide surveillance across the horizon, the building housing the entire system and supporting the antenna arrays is constructed in the shape of a triangle. The two building faces supporting the arrays, each covering 120 degrees, will monitor 240 degrees of azimuth. The array faces are also tilted back 20 degrees to allow for an elevation deflection from three to 85 degrees above the horizontal.

The radar system is capable of detecting and monitoring a great number of targets that would be consistent with a massive SLBM attack. The system must rapidly discriminate between vehicle types, calculating their launch and impact points in addition to the scheduling, data processing and communications requirements. The operation is entirely automatic, requiring people only for monitoring, maintenance and as a final check on the validity of warnings. Three different computers communicate with each other form the heart of the system, which relays the information to Cheyenne Mountain AS.

\section{Cashing In On Buried Treasure}

The purpose of this paper is to highlight the value of Early Warning and Space Surveillance Radar systems as tremendous resources as Infosphere Information Sources. The earlier mentioned Air Force directive for C2ISR mentioned the need to utilize existing Space assets. There seems to be a growing interest in new assets, but a diminishing interest in existing assets. The futuristic Space Based Radar System and the growing number of satellite links certainly promises expanded Infosphere performance and access opportunities. But as in any venture, it is important to evaluate the resources that you have in hand. 
As mentioned earlier, the most important enabler of the Global Information Grid is the Information Sources. The infrastructure to handle information is important, and appears to be getting much of the spotlight, but that structure will fall down if it does have good information. Early Warning Radar Systems such as PARCS and PAVE PAWS, and Space Surveillance Radars, such as the Eglin AFB Radar, are excellent sources of information. These systems are called Buried Treasure, because they already exist as National Information Source assets, but their full potential and value is greatly under utilized.

There are at least two powerful reasons that these ground based radar assets are highly valuable as Information Sources. First is the proliferation of nuclear weapons throughout the world along with the increased world access to long range missiles. The need for 24 hour a day 365 day a year early warning sentries increases daily with this Global Threat. Early Warning Radars were created, and created with tremendous capability, to provide this capability. Second, with the increased world interest in space assets, there also exists a need to inventory, and assess status of the over 60,000 (and growing) items in space. An item as small as a bolt can render a billion dollar satellite useless in space. Space Surveillance Radars have this inventory problem covered.

Space applications have two views. Looking down from space to Earth (or some other Planet). This view captures most people's attention. These types of assets can be positioned to focus sensors at a very specific area on the Earth's surface, and provide critical information about point targets. Their vulnerability is in the amount of energy that they can focus on their targets. Their solutions, by necessity must be very focused. The other view is that looking into space. Ground based systems have no energy limitations. They can projects enormous power to space, thus enabling them to cover large areas of space with incredible detail on targets of interest. The amazing part is that we already own the ground-based capabilities. We need only fully utilize them. Though there is much needed to give complete coverage, these existing systems can still greatly help.

\section{Infrastructure Needs}

Early Warning Radars and Space Surveillance Radars exist as National treasures for the Global Information Grid. Unfortunately, they have been greatly ignored when it comes to their upgrades and maintenance. The tremendous engineering that went into these systems, along with dedicated people from Air Force Space Command and Air Force Electronic Systems Command have kept these systems up and running well past their original expected lives. The capability still exist, and as discussed is still greatly needed, but needs some long awaited capitol improvements. Some of these systems are still operating with their original 1960s vintage computer systems.

Recently much National attention was focused on the eroding condition of our infrastructure of bridges. A ready source of capitol (gasoline taxes) has been leveraged to address this situation, and the bridges are steadily being repaired or replace. No gasoline tax exists to address ground-based radars. Military legacy system sustainment revenues

are in short supply. Ground-based radar systems continue to be low on the priority list for 
available funds. Perhaps, if the global information consumer, including the C2ISR community, better understood the value of these systems this situation could be reversed.

\section{References}

(1) United States Air Force Scientific Advisory Board Report on "Building the Joint Battlespace Infosphere”, Volume 1: Summary, SAB-TR-99-02, December 17,1999.

(2) United States Air Force Aerospace Command Control Intelligence, Reconnaissance (C2ISR) Campaign Plan 2000, December 23, 1999.

(3) Navathe, S. B., A Knowledge-Based Approach to Integrating and Querying Distributed Information Systems Heterogeneous Intelligent Processing for Engineering Design (HIPED, Air Force Research Laboratory Final Report \# WL-TR-97-1165, August 1997.

(4) Air Force Space Command's Home Page, http://www.spacecom.af.mil/hqafspc, 7 April 2000. 